\title{
Claros, le temple d'Apollon : travaux réalisés en 2014
}

Jean-Charles Moretti, Nicolas Bresch, Isabel Bonora, Jean-Jacques Malmary et Olivier Riss

\section{OpenEdition \\ 12 Journals}

\section{Édition électronique}

URL : http://journals.openedition.org/anatoliaantiqua/364

DOI : 10.4000/anatoliaantiqua.364

\section{Éditeur}

IFEA

Édition imprimée

Date de publication : 1 juin 2015

Pagination : 291-300

ISBN : 9782362450600

ISSN : 1018-1946

\section{Référence électronique}

Jean-Charles Moretti, Nicolas Bresch, Isabel Bonora, Jean-Jacques Malmary et Olivier Riss, «Claros, le temple d'Apollon : travaux réalisés en 2014 », Anatolia Antiqua [En ligne], XXIII | 2015, mis en ligne le 30 juin 2018, consulté le 18 décembre 2020. URL : http://journals.openedition.org/anatoliaantiqua/ 364 ; DOI : https://doi.org/10.4000/anatoliaantiqua.364 


\title{
ANATOLIA ANTIQUA ESKI ANADOLU
}

\author{
XXIII
}

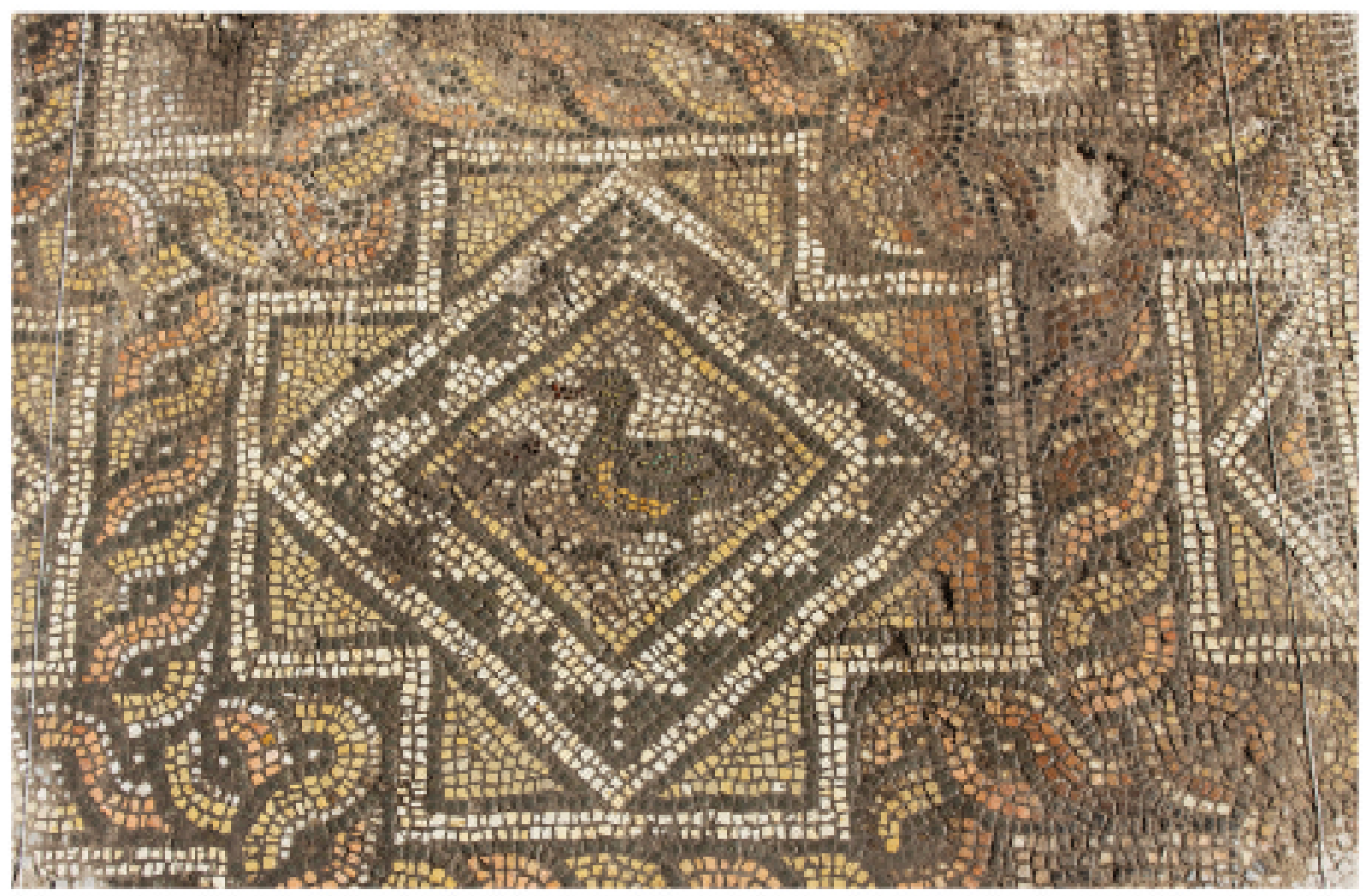

INSTITUT FRANC̣AIS D'ETUDES ANATOLIENNES GEORGES-DUMEZIL CNRS USR 3131 


\section{TABLE DES MATIERES}

Hélène BOUILLON,

On the anatolian origins of some Late Bronze egyptian vessel forms

Agneta FRECCERO,

Marble trade in Antiquity. Looking at Labraunda

Şehnaz ERASLAN,

Dionysus and Ariadne in the light of Antiocheia and Zeugma Mosaics

Ergün LAFLI et Gülseren KAN ŞAHIN,

Middle Byzantine ceramics from Southwestern Paphlagonia

Mustafa AKASLAN, Doğan DEMIRCİ et Özgür PERÇİN en collaboration avec Guy LABARRE, L'église paléochrétienne de Bindeos (Pisidie)

Anaïs LAMESA,

La chapelle des Donateurs à Soğanlı, nouvelle fondation de la famille des Sképidès

Martine ASSENAT et Antoine PEREZ,

Localisation et chronologie des moulins hydrauliques d'Amida. A propos d'Ammien Marcellin,

XVIII, 8,11

Helke KAMMERER-GROTHAUS,

$»$ Ubi Troia fuit«

Atzik-Köy - Eine Theorie von Heinrich Nikolaus Ulrichs (1843)

CHRONIQUES DES TRAVAUX ARCHEOLOGIQUES EN TURQUIE, 2015

Sami PATACI et Ergün LAFLI,

Surveys in Ardahan on the turkish-georgian borderline in 2013 and 2014

Çĭ̆dem MANER,

Preliminary report on the second season of the Konya-Ereğli survey (KEYAR) 2014

Dominique BEYER, Can KARAVUL, Françoise LAROCHE-TRAUNECKER et Aksel TiBBET,

Rapport préliminaire sur les travaux de la mission archéologique de Zeyve Höyük-Porsuk 2014

Jean-Charles MORETTI avec la collaboration de Nicolas BRESCH, Isabel BONORA,

Jean-Jacques MALMARY et Olivier RISS,

Claros, le temple d'Apollon : travaux réalisés en 2014

Olivier HENRY et Erika ANDERSSON, Christophe BOST, Ömür Dünya ÇAKMAKLI, Angela COMMITO, Mélissa CORMIER-HUGUET, Peter DE STAEBLER, Pierre DUPONT, Duygu ERGENÇ, Axel FREJMAN, Banu KEPENEK, Pascal LEBOUTEILLER, Haral NILSSON, Felipe ROJAS, Baptiste VERGNAUD, 


\author{
Jean-Charles MORETTI* \\ avec la collaboration de Nicolas BRESCH ${ }^{* *}$, Isabel BONORA ${ }^{* * *}$, \\ Jean-Jacques MALMARY**** et Olivier RISS ${ }^{* * * * *}$
}

\title{
CLAROS, LE TEMPLE D'APOLLON : TRAVAUX REALISES EN 2014
}

La campagne de 2014 a duré cinq semaines sur le terrain pendant lesquelles N. Şahin, professeur à l'université d'Egée à Izmir, directrice de la mission turque de Claros, nous a offert les meilleures conditions de travail. Le plan du temple qui avait été réalisé dans les années 1950 par P. Bonnard et qui, jusqu'à présent, n'avait été l'objet que de corrections limitées par G. Charpentier, a été refait sur la base de mesures prises sur le monument, de levés topographiques réalisés l'année dernière avec une station totale ${ }^{1}$ et de relevés photogrammétriques faits cette année pour des détails de la crypte. Pour les fondations du dallage du ptéron, le relevé de P. Bonnard a servi de base au nouveau plan. Nous disposons dorénavant d'un plan fiable et complet du temple sur support numérique (Fig. 1). Plusieurs coupes et élévations ont aussi été réalisées cette année (Fig. 2) et nous avons travaillé en parallèle d'une part sur le projet du temple mis en chantier à la fin du $4^{\mathrm{e}} \mathrm{s}$., reprenant, synthétisant et approfondissant certaines observations faites ces dernières années, et d'autre part sur certains éléments de l'édifice de la fin de l'époque hellénistique et de l'époque impériale : la base de la statue d'Octavien qui se trouvait dans le naos et l'entablement de la péristasis. Le premier volet de ce programme a débouché sur la rédaction d'un article qui paraîtra prochainement. Il conduit à proposer le plan de la (Fig. 3) pour le projet de temple à cour mis en chantier vers 300 av. J.-C. et abandonné au moment de la prise de Colophon par Lysimaque, en 295 probablement. Des indices ont été identifiés qui rendent très probable la restitution d'un large escalier d'accès à la cour se développant dans l'épaisseur du mur de refend. Nous rendons compte ci-dessous du second volet du programme.

\section{LA BASE DE LA STATUE D'OCTAVIEN}

En 1955 fut découvert dans la salle des consultants, avec plusieurs fragments des statues de la triade apollinienne, un bloc qui, l'année suivante, se révéla appartenir à la base d'une statue d'Octavien'2. L'inscription fut signalée par L. Robert ${ }^{3}$ et publiée par J.-L. Ferrary ${ }^{4}$. Elle date des années 31-28. Nos prédécesseurs se sont peu intéressés au bloc qui porte la dédicace et n'ont pas remarqué qu'il s'agissait d'une base remployée à l'envers qui avait porté une autre inscription et une autre statue (Fig. 4 et 5). En voici une description :

Marbre blanc. Manquent plus de la moitié du premier lit d'attente et les angles du premier lit de pose.

Face A (première face postérieure, seconde face antérieure) : dressée au ciseau grain d'orge. Dans sa partie supérieure, elle porte l'inscription :

*) IRAA, CNRS, MSH MOM, Université Lumière Lyon 2. Mission financée par le Ministère des Affaires étrangères et du Développement international et, pour un projet intitulé "A Shipwrecked Ancient Marble Column Destined for the Temple of Apollo at Claros", par le Partner University Fund (PUF) et l'Andrew W. Mellon Foundation in the field of Humanities. Outre les signataires de cette chronique, I. M. Erker (étudiante à l'ENSAS) et D. Laroche (ENSAS) ont participé ponctuellement à la campagne.

**) Architecte, IRAA, CNRS

***) Musée du Louvre.

****) Architecte, IRAA, CNRS.

$* * * * *)$ Architecte.

1) Moretti et al. $2014: 237$.

2) Martin, Carnets Claros 1956, 13 septembre.

3) Robert $1974: 155$.

4) Ferrary $2000: 357-359$. 


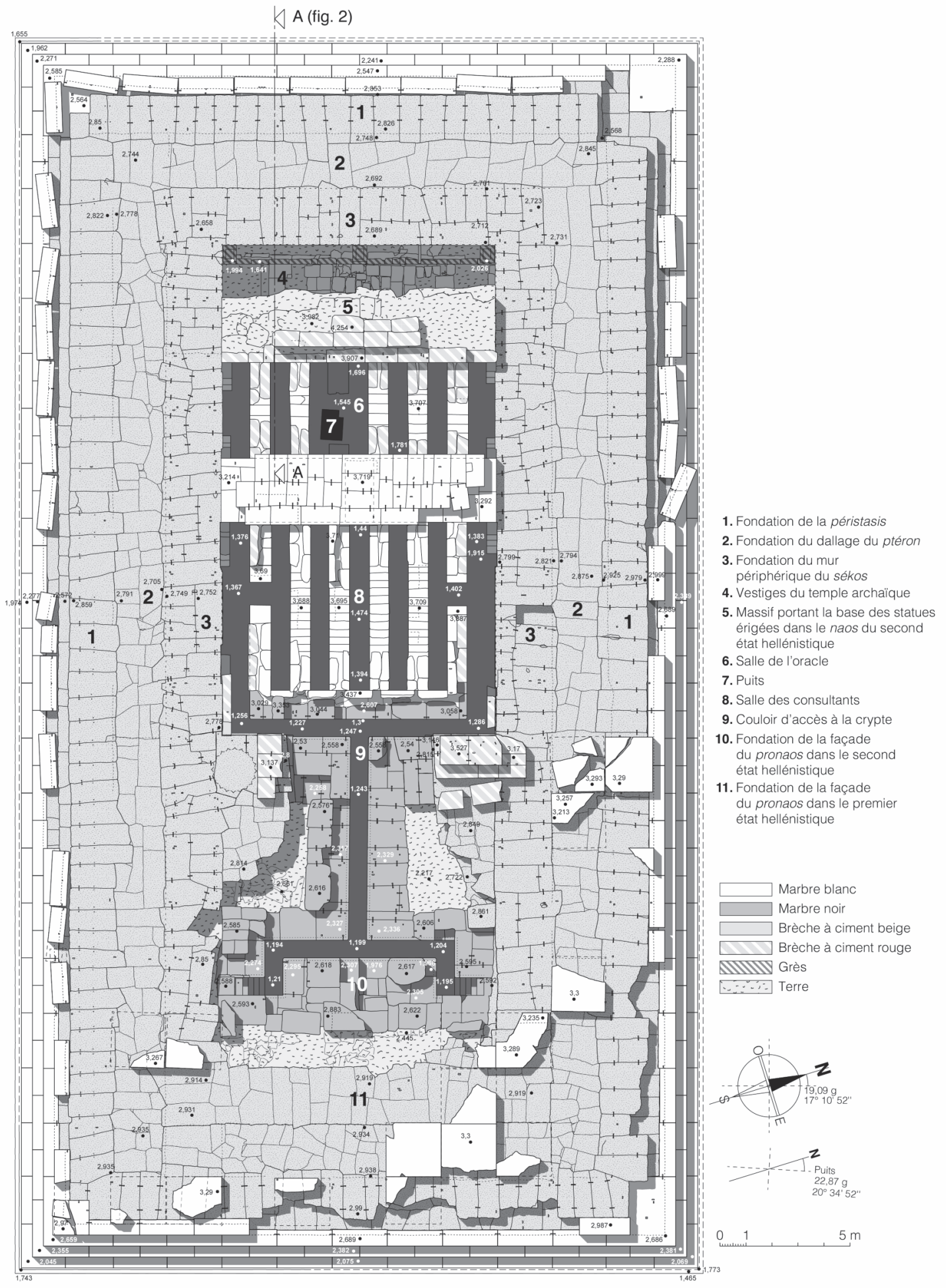

Fig. 1 : Plan général des vestiges du temple au 1/250 (J.-J. Malmary). 


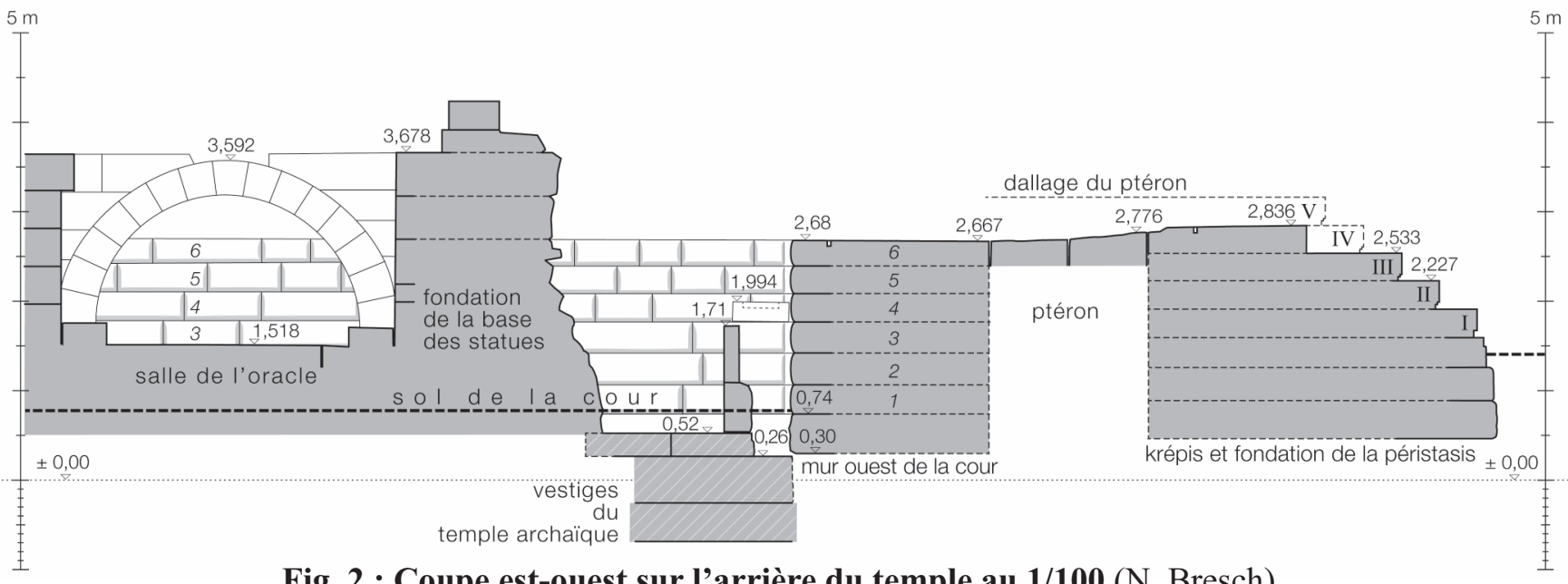

Fig. 2 : Coupe est-ouest sur l'arrière du temple au 1/100 (N. Bresch). L'emplacement de la coupe est indiqué sur la Fig. 1 (coupe A-A).

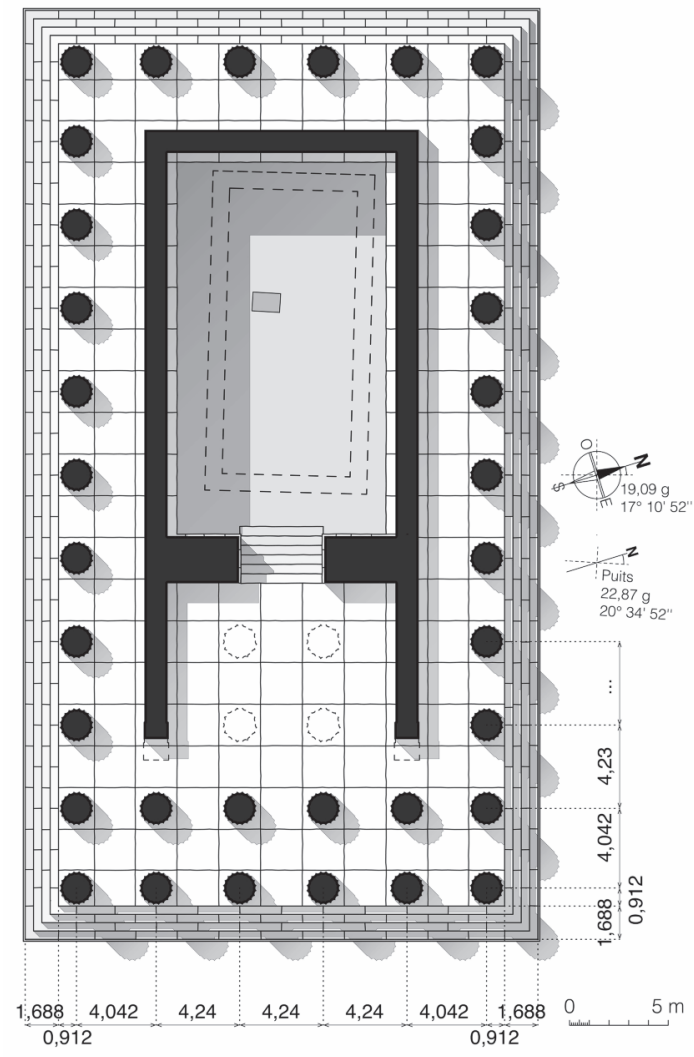

Fig. 3 : Proposition de restitution du plan projet initial du temple hellénistique au 1/500 (J.-J. Malmary).

L'emplacement approximatif des murs de la cour du temple archaïque est indiqué par des traits discontinus.
$\mathrm{O} \delta \tilde{\eta} \mu \mathrm{s}$

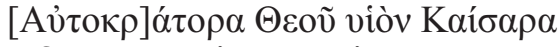

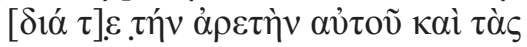

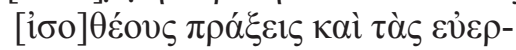

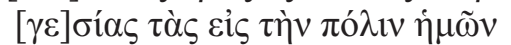

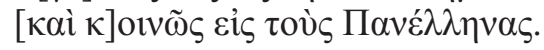

Ht. des lettres : 2,5 cm. Ecriture peu soignée. Les deux premières lignes, qui peuvent se comprendre seules, sont gravées plus profondément que les autres. Ce travail a peut-être été fait dans un second temps. Le style des lettres et leur taille sont les mêmes dans toutes les lignes.

Face B (face de joint gauche) mal conservée. Reste une partie d'un mince bandeau d'anathyrose le long de la face E.

Face C (premier lit d'attente, second lit de pose) : dans la partie médiane, encastrement pour le tenon d'un pied de statue en bronze. Une autre mortaise rectangulaire, incomplètement conservée, lui est associée à gauche. En bordure de l'arête de la face $\mathrm{D}$, mortaise en queue d'aronde pour agrafe en bois. Trace possible d'une autre mortaise pour un pied dans l'angle postérieur gauche.

Face D (face de joint droit) : mince bandeau d'anathyrose en $p i$.

Face $\mathbf{E}$ (première face antérieure, seconde face postérieure) : sur une bande large au sommet et plus fine en bordure de l'arête de droite, vestiges de la surface originelle, finement dressée au ciseau grain d'orge. Dans la bande supérieure, on lit encore trois lettres hautes de $3 \mathrm{~cm}: \Pi I \Sigma$ (Fig. 6). Le pi a la haste verticale de droite plus courte que celle de gauche ; le iota est doté d'apices comme le sigma, qui a les branches supérieure et inférieure légèrement concaves. 


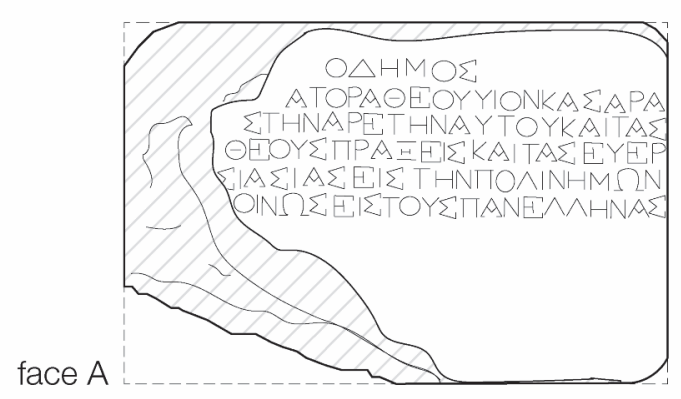

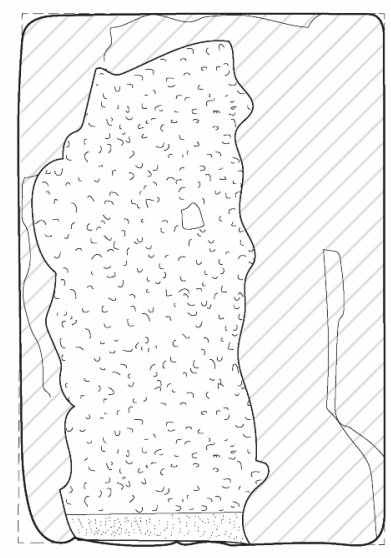

face $B$

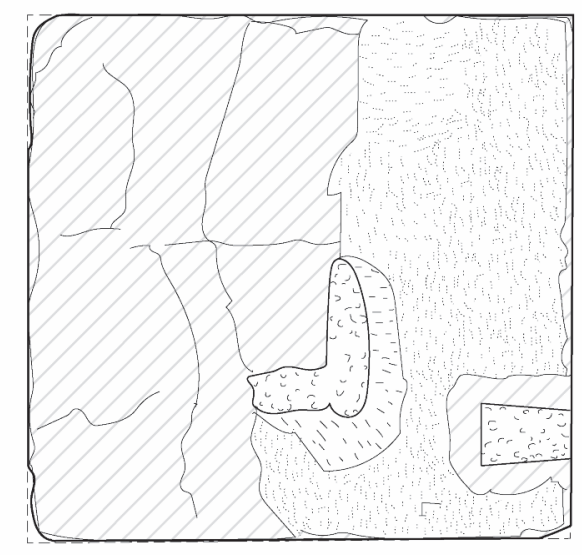

face $\mathrm{C}$

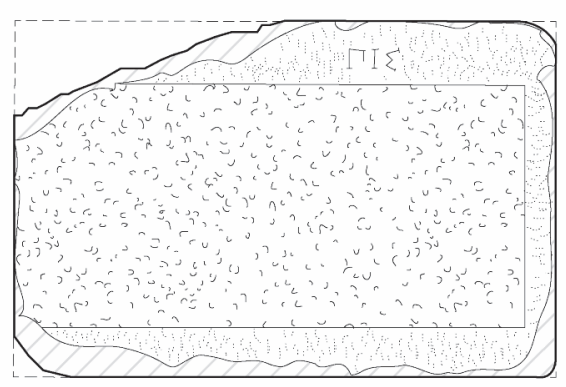

face $\mathrm{E}$

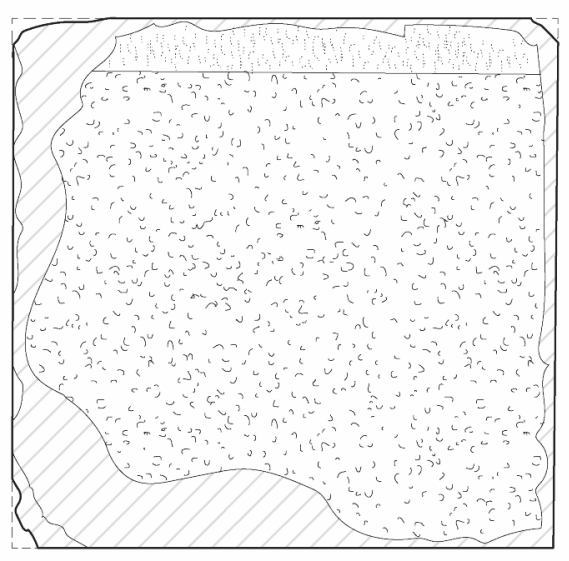

face $\mathrm{F}$

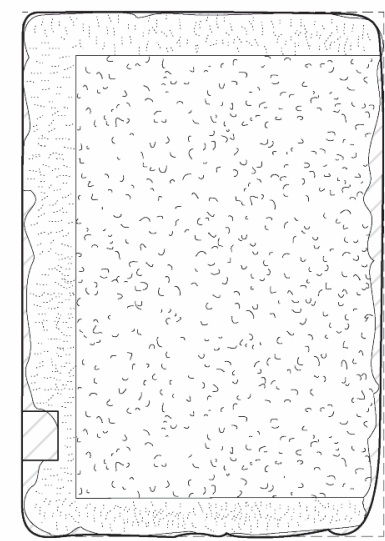

face $D$

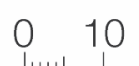

60

$120 \mathrm{~cm}$

Fig. 4 : La base de la statue d'Octavien, relevé au 1/20 (I. Erker). 


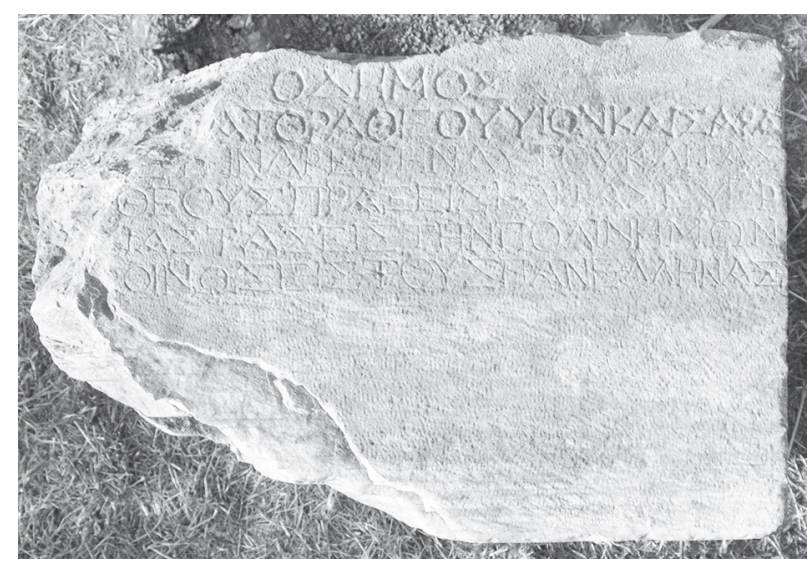

Face A

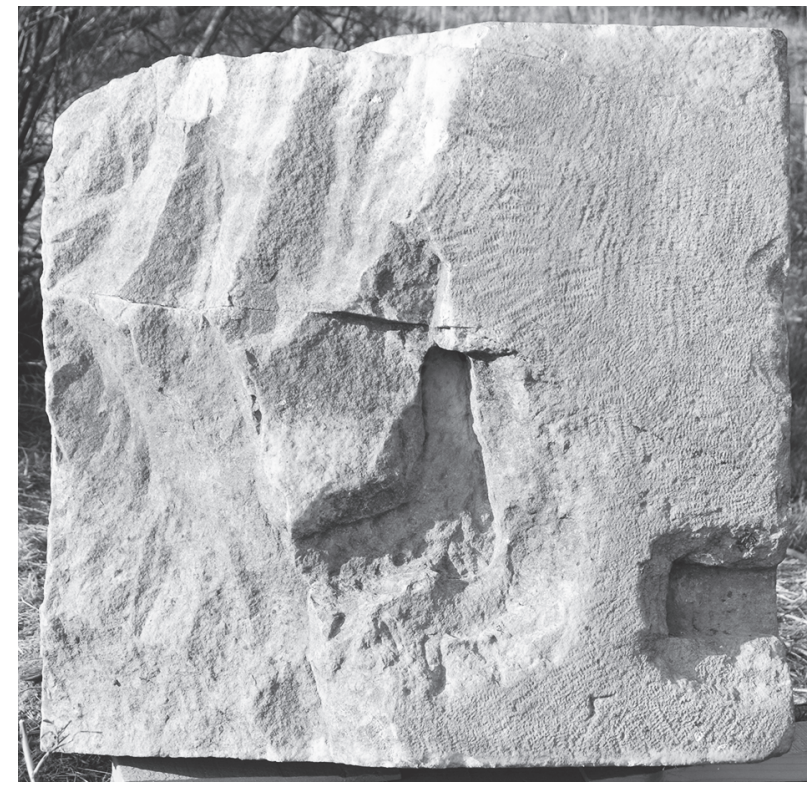

Face C

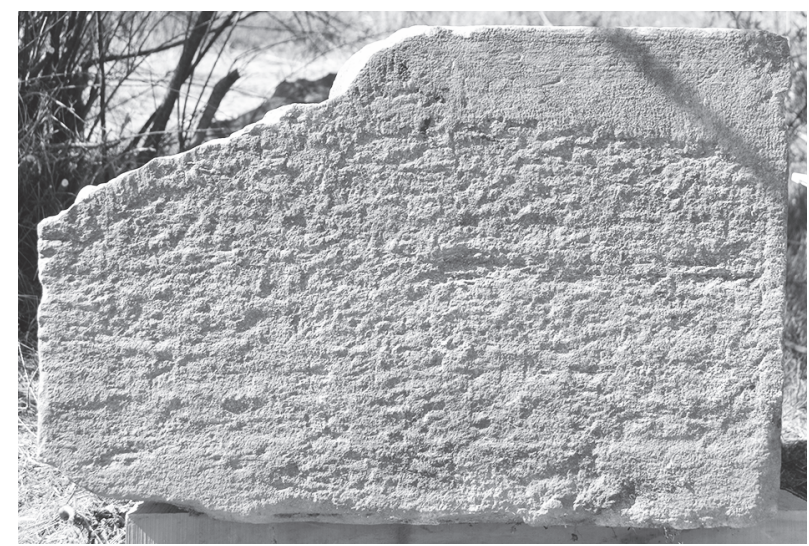

Face $\mathbf{E}$

Fig. 5 : La base de la statue d'Octavien : faces A, C et $\mathbf{E}$ (clichés J.-Ch. Moretti).
L'écriture conviendrait au $3^{\text {e }}$ s. ${ }^{5}$. Le reste de la surface a été ravalé au ciseau droit pour faire disparaître l'inscription. Il n'en demeure que quelques hastes.

Face F (premier lit de pose, second lit d'attente) : bandeau d'anathyrose en bordure de la face $\mathrm{E}$.

Dans une première phase, le bloc a sans doute fait partie d'une base composée d'au moins trois blocs. Seul le bloc central, retourné, a été retenu pour la statue d'Octavien. Lors du remploi, la première inscription a été ravalée et la seconde centrée sur l'ancienne face postérieure. La présence d'une assise de couronnement non scellée portant la statue d'Octavien paraît assurée. Celle d'une assise avec une moulure de base est possible.

\section{L'ENTABLEMENT DE LA PERISTASIS}

Les blocs provenant de l'entablement de la péristasis mis en place aux frais d'Hadrien avaient déjà fait l'objet d'un premier inventaire ces dernières années qui avait débouché sur une restitution générique de l'ordre. Le travail de 2014 a eu pour objectif de déterminer l'extension de cet entablement car il ne convient pas d'admettre a priori qu'il a recouvert toutes les colonnes érigées, probablement à la fin de l'époque hellénistique, soit les six colonnes de la façade et cinq sur chacun des longs côtés. En l'absence de plan marquant l'emplacement des blocs au moment de leur découverte, il nous a fallu travailler à partir des photographies prises pendant la fouille par R. Martin (Fig. 7). L'identification sur ces photographies de plusieurs blocs provenant de l'entablement permet dans l'état actuel de nos recherches de proposer la répartition suivante entre les faces sud, est et nord.

\section{La face sud (Fig. 8)}

Peu de blocs de l'entablement de la face sud sont conservés :

- Peut-être rien de l'architrave : le seul candidat possible est le bloc 1666, qui peut provenir de la face sud ou de la face nord.

- Trois voire quatre blocs de frise. La provenance est assurée pour le bloc d'angle 171, une pièce à triglyphe et métope à droite $\mathbf{1 3 6}+\mathbf{1 3 7}$ et le dernier bloc de la frise vers l'Ouest 1698. Elle ne l'est pas pour la pièce à triglyphe et métope à droite $\mathbf{1 6 9 9}$, qui peut provenir de la face sud ou de la face est.

- Deux voire trois blocs de corniche. La provenance de 138, qui n'était pas couvert et qui avait une voie ornée d'un foudre, est assurée tout comme 


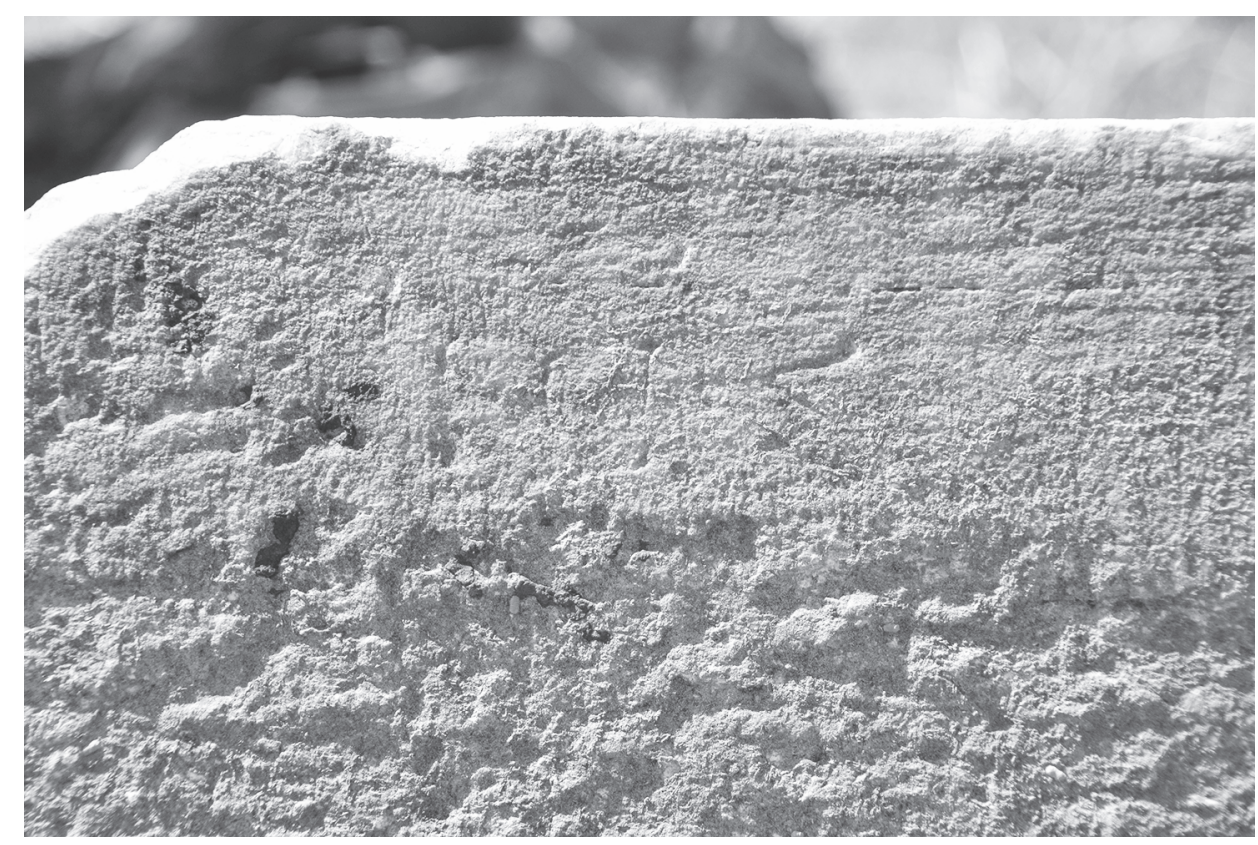

Fig. 6 : La base de la statue d'Octavien : vestige de l'inscription sur la face antérieure originelle (face E) (cliché J.-Ch. Moretti).

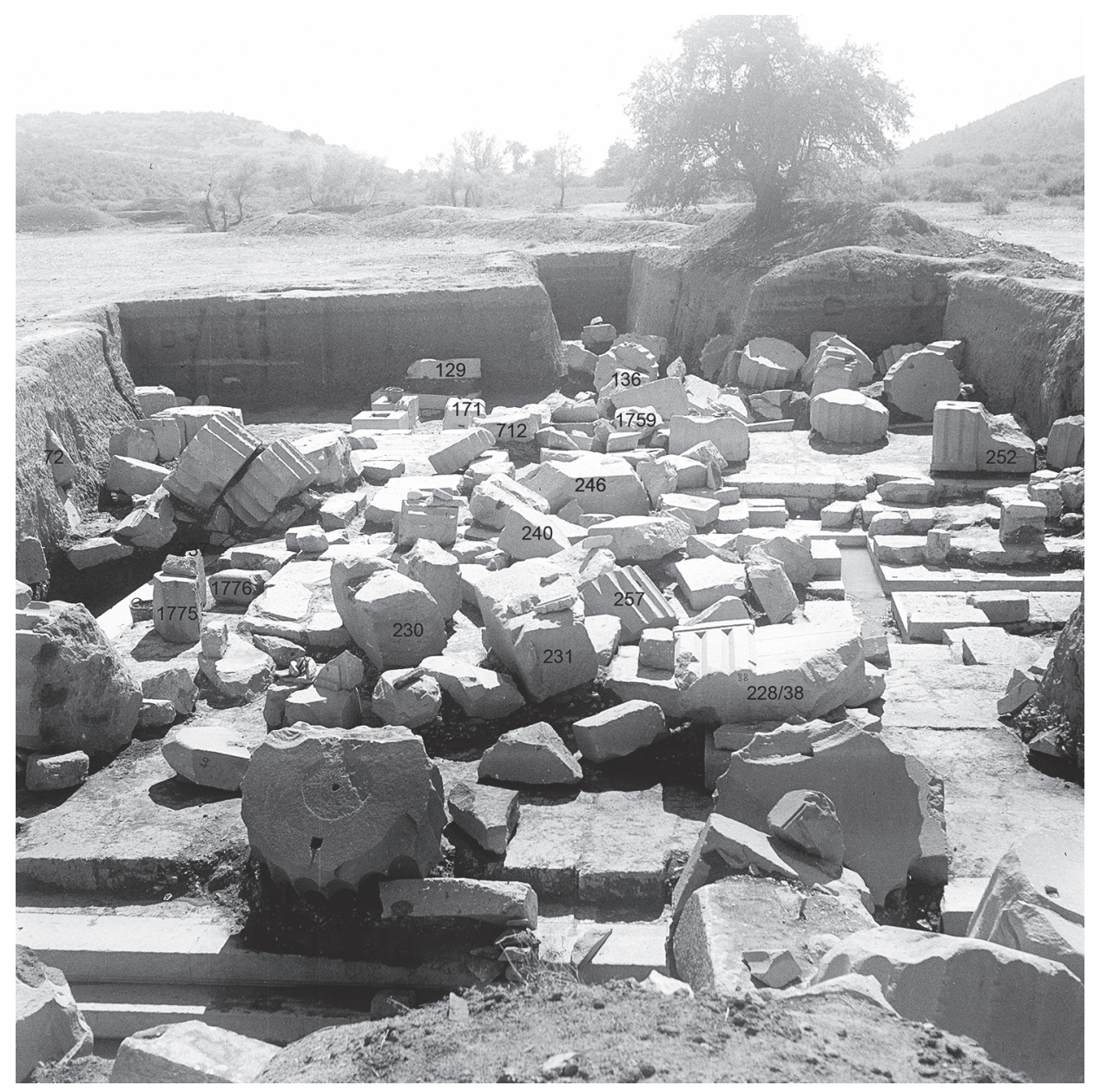

Fig. 7 : Blocs identifiés sur un cliché pris par

R. Martin pendant la fouille du temple. 


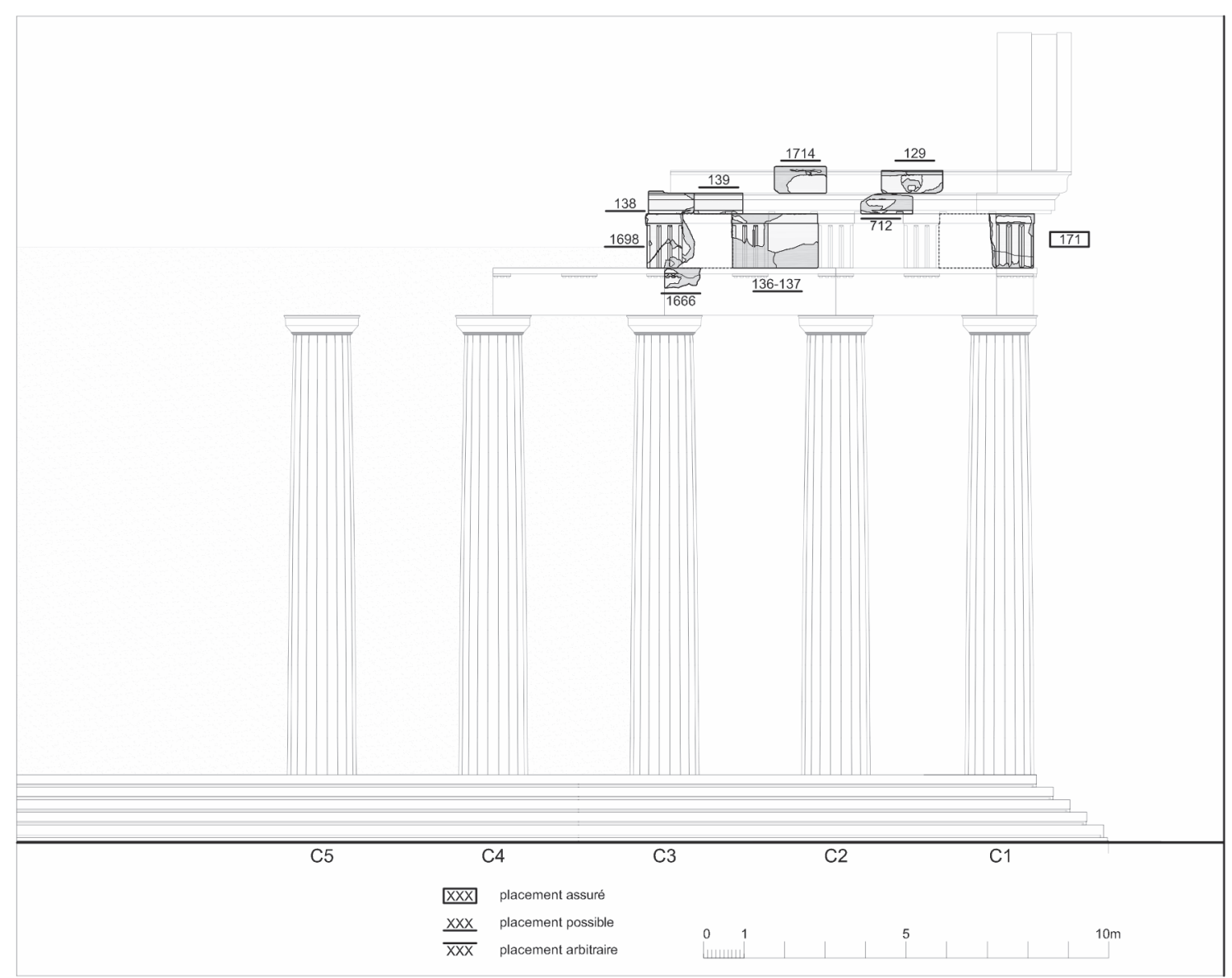

Fig. 8 : Restitution de l'entablement de la face sud du temple au 1/200 (I. Bonora et O. Riss).

celle de $\mathbf{1 3 9}$, ce qui n'est pas le cas de celle de $\mathbf{7 1 2}$, qui pourrait aussi provenir de la façade est.

- Deux blocs de sima (1714 et 129), dont un est orné d'une tête de lion (129).

Les blocs de corniche $\mathbf{1 3 8}$ et $\mathbf{1 3 9}$ ont été mis au jour côte à côte au pied de la troisième colonne à compter de l'angle sud-est (C3). Leur lieu de découverte et le rythme de leurs mutules avaient déjà conduit $\mathrm{P}$. Bonnard à les associer. Leur restitution au-dessus de la colonne au pied de laquelle ils ont été trouvés s'impose, mais il est difficile de savoir si le mutule de $\mathbf{1 3 8}$ couronnait le triglyphe placé sur l'axe de la colonne ou la métope située entre la troisième et la quatrième colonne. Dans la première hypothèse, illustrée par la (Fig. 8), le triglyphe 1698, qui était le dernier bloc de frise, est restitué audessus de la troisième colonne et est couronné par le dernier bloc de corniche 138. Dans la seconde, le triglyphe se trouverait sur l'entraxe entre la troisième (C3) et la quatrième colonne $(\mathrm{C} 4)$, voire plus à l'Ouest encore et il n'aurait pas été couvert par une corniche.

\section{La façade est (Fig. 9)}

L'entablement de la façade, le seul qui ait été entièrement achevé, est le mieux conservé. De l'entablement horizontal, il demeure :

- Quatre éléments d'architrave dont la restitution de l'emplacement est assurée par l'inscription qu'ils portent $(69,72,1997,328+M 276)$.

- Six, au moins, de frise : 171, 97, 240, M34, 237, M84. Il s'y ajoute peut-être 1699, qui, nous l'avons noté, provient de la face est ou de la sud, ainsi que M6 et M7 qui sont visibles sur des photographies près de l'angle nord-est de la krépis et peuvent provenir de la face est ou de la face nord.

- Au moins un bloc de corniche (58), mais le doute existe pour plusieurs autres : 712, M316, 1207, 1761 et 1778.

Du fronton, il demeure :

- le bloc de l'angle nord-est qui combine corniche et sima (114); née ; 


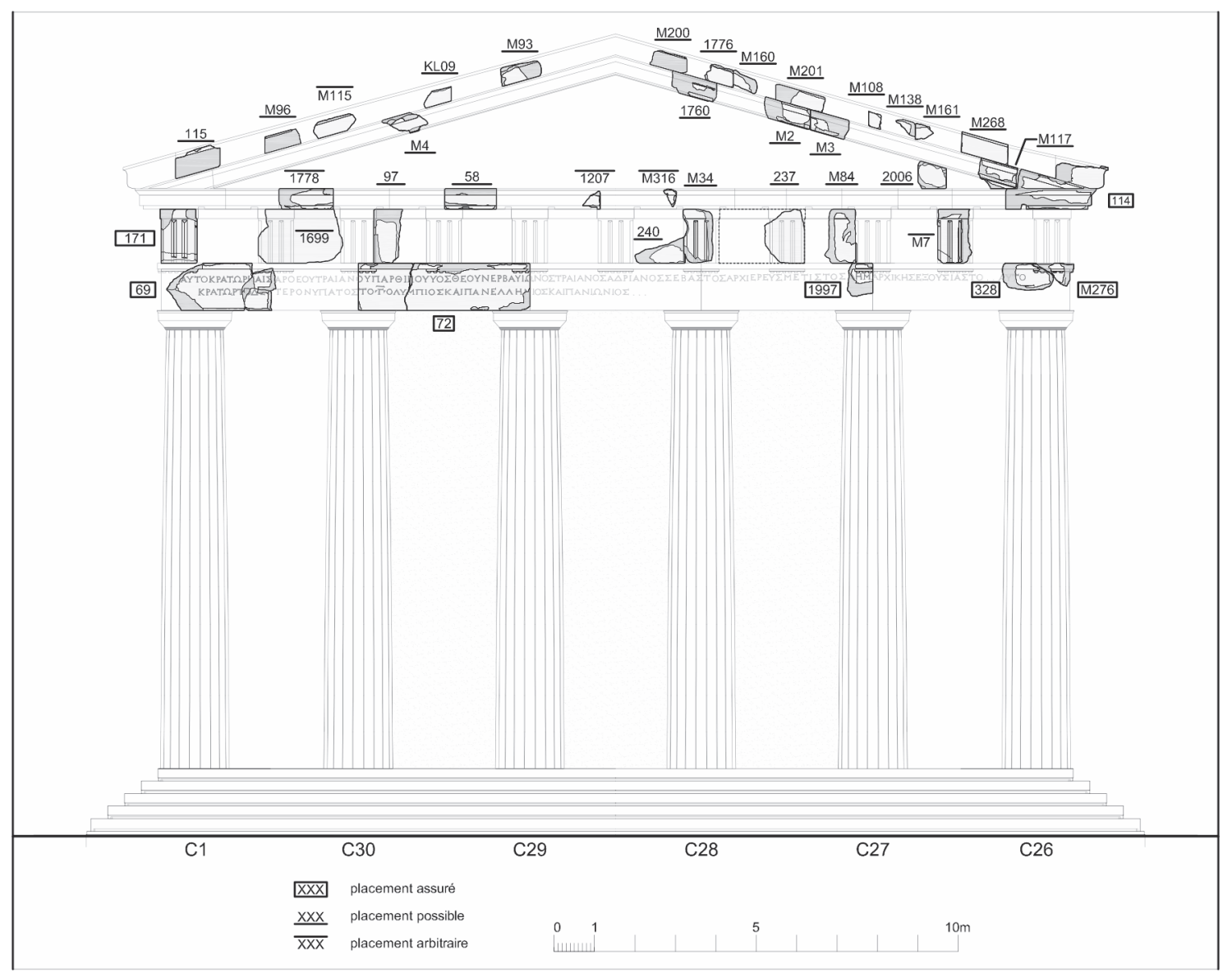

Fig. 9 : Restitution de l'entablement de la face est du temple au 1/200 (I. Bonora et O. Riss).

- cinq blocs de la corniche rampante (à droite ; M/117, M/2, M/3, 1760 ; indéterminé : M/4)

- quatorze blocs de la sima rampante : quatre du rampant sud (M93, M96, 115, KL09), neuf du rampant nord (M268, M108, M160, M161, M200, M201, 1776, M138) et un indéterminé (M115).

L'emplacement du bloc d'angle du fronton $\mathbf{1 1 4}$ et de celui du bloc de tympan 2006 sont assurés, tout comme celui du triglyphe d'angle sud-est (171). Pour le reste, au-dessus de l'architrave, aucun emplacement ne peut être restitué avec certitude. Nous nous sommes fiés aux positions de chute, pour placer M268 et M161 au-dessus du premier entrecolonnement à compter du Nord (entre C26 et C27) et $\mathbf{1 7 7 6}$ au droit de la colonne C28.

\section{La face nord (Fig. 10)}

L'entablement de la face nord est le plus mal conservé.

- De l'architrave, rien n'est sûr en dehors du petit côté de M276. Lors des déplacements récents, deux fragments ont été enregistrés comme provenant du Nord : M111 et M171. Le bloc 1666, nous l'avons noté, peut provenir du Nord ou du Sud.

- Les deux triglyphes M6 et M7 peuvent provenir de la face est ou de la face nord, mais aucun d'eux n'est un triglyphe d'angle.

- L'origine de la corniche horizontale M/175 est assurée : elle a été découverte légèrement au Nord de la troisième colonne de la face nord. La corniche 1761, qui est actuellement rangée à l'angle nord-est du temple, pourrait aussi provenir de la face nord, ainsi que d'autres fragments de provenance inconnue (M316, 1207, 1761 et 1778).

- Aucune sima ne peut être assurément restituée à la face nord.

Rien, semble-t-il, n'empêche donc de penser que l'entablement des longs côtés n'a pas été construit à l'Ouest de la troisième colonne $(\mathrm{C} 24)$, qui était alignée sur la façade du pronaos. Ainsi s'expliquerait que les tambours des colonnes 4 et 5 des deux longs côtés (C4-C5 au Sud, C22-23 au Nord) n'aient recouvert aucun bloc d'entablement quand ils ont été abattus. Pour la destruction, on a d'abord fait tomber 


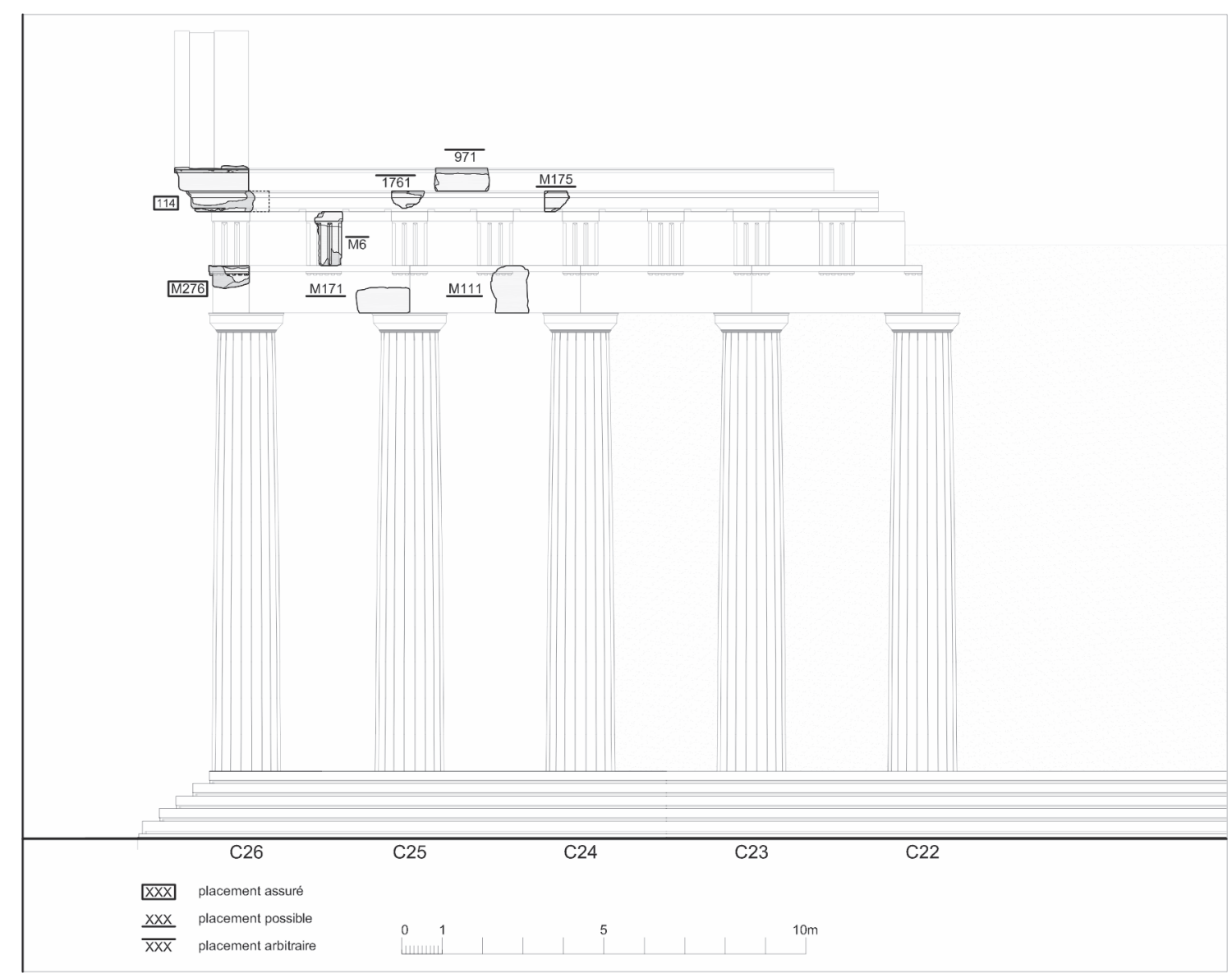

Fig. 10 : Restitution de l'entablement de la face nord du temple au 1/200 (I. Bonora et O. Riss).

l'entablement, puis on en a récupéré certains éléments : les architraves et les métopes, principalement, ainsi que le tympan en façade. Les colonnes ont été abattues ensuite pour prendre le métal des scellements, déjà récupéré par creusement dans les joints des tambours inférieurs. Très peu de tambours abattus ont été emportés ou retaillés. L'étude des chapiteaux prévue pour l'année prochaine permettra sans doute de préciser ces restitutions.

L'année 2014 a ainsi permis des avancées notoires dans la connaissance du temple d'Apollon, mais aussi dans la rédaction de la publication monographique en préparation. L'étude de la première phase du temple hellénistique est dorénavant achevée ainsi que l'analyse comparative qui permet de situer l'édifice dans la renaissance ionienne. L'étude de la base de la statue d'Octavien, chichement réa- lisée dans un bloc de remploi mal ravalé, a montré à sa manière que Colophon vers 30 av. J.-C. n'était pas une cité prospère. Celle de tous les blocs de l'entablement de la péristasis a conduit à réviser à la baisse la donation d'Hadrien, qui a seulement permis d'ériger une partie de l'entablement et le fronton de la façade sur des colonnes qui étaient sans doute déjà en place à la fin de l'époque hellénistique.

2014 a aussi été marquée par la parution des actes du colloque sur Claros que nous avions organisé à Lyon en 2012 : J.-Ch. Moretti, éd., avec la collaboration de L. Rabatel, Le sanctuaire de Claros et son oracle, Actes du colloque international de Lyon, 13-14 janvier 2012, TMO 65, Lyon, 2014.

J.-Ch. M. et al. 


\section{BIBLIOGRAPHIE}

Moretti, J.-Ch. et al., 2014 : J.-Ch. Moretti avec la collaboration de N. Bresch I. Bonora, J.-J. Malmary et O. Riss, "Claros, le temple d'Apollon : travaux réalisés en 2013", Anatolia Antiqua XXII : 237-246.

Ferrary, J.-L., 2000 : "Les inscriptions du sanctuaire de Claros en l'honneur de Romains", BCH 124 : 331-376.
Robert, L., 1974 : “Rapports sur les cours à l’Ecole des Hautes Etudes et au Collège de France et sur les missions", Opera Minora Selecta IV, Amsterdam : 63-364. 


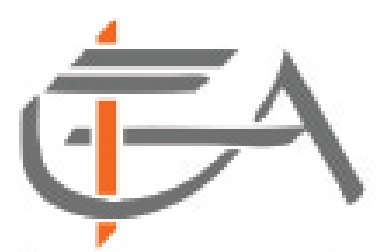

Institut Français d'Etudes Anatoliennes

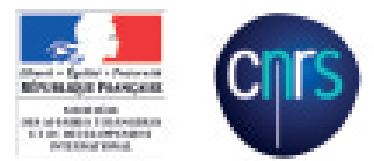

Eglise paléochrétienne de Bindéos (Pisidie), détail de la mosaïque ornant le sal de la nef centrale, $4^{e} \mathrm{~s}$. ap. J.-C. 